\title{
Akute respiratorische Insuffizienz bei schwerer Lungenschädigung - ARDS und ALI
}

\author{
Acute Respiratory Insufficiency due to Severe Lung Injury - ARDS and ALI
}

Autor

Institute
M. Pfeifer ${ }^{1,2}$

Klinik Donaustauf, Zentrum für Pneumologie und Beatmungsmedizin, Donaustauf

2 Klinik und Poliklinik für Innere Medizin II, Pneumologie, Klinikum der Universität Regensburg, Regensburg eingereicht 21.7 .2010

akzeptiert 22.7.2010

Bibliografie

Dol http://dx.doi.org/

10.1055/s-0030-1255680

Pneumologie 2010; 64:

590-594 @ Georg Thieme

Verlag KG Stuttgart · New York ISSN 0934-8387

\section{Korrespondenzadresse Prof. Dr. Michael Pfeifer Klinik Donaustauf Zentrum für Pneumologie und Beatmungsmedizin Ludwigstraße 68 93093 Donaustauf michael.pfeifer@ klinik.uni-regensburg.de}

\section{Zusammenfassung \\ $\nabla$}

Das Acute-Respiratory-Distress-Syndrom (ARDS) als schwerste Form des akuten Lungenschadens (acute lung injury - ALI) wurde mit der frühen Entwicklung der Intensiv- und Beatmungsmedizin als eigene Krankheitsentität beschrieben. In den 4 Jahrzehnten seit der Erstbeschreibung wuchs das Wissen über Ätiologie, Physiologie, Histologie und Epidemiologie dieser oft tödlichen pulmonalen Komplikation einer schweren akuten Erkrankung wie Pneumonie oder Sepsis. Die wesentlichen therapeutischen Fortschritte waren anfänglich nur durch die Verbesserung der intensivmedizinischen Methoden und Überwachungssysteme zu erreichen. Die große ARDS-NetworkStudie zur Höhe des Atemzugsvolumens unter Beatmung zeigte dann eindrucksvoll, dass gezielte klinische Studien auch beim ARDS durchführbar sind und wesentliche Erkenntnisse liefern. Diese Studie, wie auch nachfolgende große ARDS-Studien, haben entscheidend die Beatmungstherapie verändert und $\mathrm{zu}$ therapeutischen Konzepten geführt, die die Prognose der Erkrankung verbessern. Eine zunehmend standardisierte Versorgung der ARDS-Patienten mit innovativen Therapieverfahren wie extrakorporale Gasaustauschssysteme werden zu einer weiteren Verbesserung der Therapiemöglichkeiten dieses schweren Krankheitsbildes führen. Die moderne Pneumologie ist hier im besonderen Maße gefordert und kann aufgrund der breiten Erfahrung in allen Bereichen der Erkrankungen der Atmungsorgane einen wesentlichen Beitrag liefern.

\section{ARDS - Geschichte einer jungen Entität $\nabla$}

100 Jahre sind für Medizin-Geschichte eine kurze Periode - für das Krankheitsbild des ARDS umfasst jedoch diese Zeitperiode nahezu jeden wichtigen Schritt zur Pathophysiologie, Therapie und Inzidenz dieser Erkrankung. Die erste Beschrei-

\section{Abstract \\ $\nabla$}

As a consequence of the novel therapeutic option of mechanical ventilation in early intensive care medicine, the acute respiratory distress syndrome (ARDS) was defined as a disease entity of its own representing the most severe form of acute lung injury (ALI). Since its first description four decades ago, our knowledge about the aetiology, physiology, histology and epidemiology of this lethal pulmonary complication of severe acute diseases such as pneumonia or sepsis has been increasing steadily. The initial major therapeutic advances were due to improvements in intensive care medical procedures and monitoring. The large ARDS Network clinical trial on the magnitude of tidal volume impressively demonstrated the feasibility of targeted clinical trials in patients with ARDS that provide robust evidence in this field. This clinical trial, as well as following large-scale trials in ARDS patients, led to significant changes of ventilation therapy and therapeutic strategies that improve the outcome of this disease entity. Advances in the standardisation of care for ARDS patients involving innovative therapeutic procedures such as extracorporeal gas exchange systems will lead to a further improvement in ARDS management and outcome. Modern pulmonary medicine can play a pivotal role in this process and can contribute its rich experiences in all areas of the respiratory system.

bung des Krankheitsbildes liegt jedoch schon länger zurück. 1821 beschreibt Laennec in seinen Abhandlungen über die Pathologie der Lunge und des Herzens eine idiopathische Anasarka der Lunge; ein Lungenödem ohne krankhafte Veränderungen des Herzens in „A treatise on disease of the chest" [1]. Mit der Verbreitung der Intensiv- 
medizin in den 50er- und 60er-Jahren des letzten Jahrhunderts und der Entwicklung der positiven Druckbeatmung über den endotrachealen Tubus nahm die Zahl der Beatmungen von Patienten mit schwerer respiratorischer Insuffizienz deutlich zu. Häufig sah man ein Lungenödem als Ursache - unterschied jedoch nicht zwischen kardialem oder nicht kardialem Lungenödem [2].

Im Rahmen der wachsenden Erfahrungen wurden dann die unter maschineller Ventilation gesehenen diffusen beidseitigen pulmonalen Infiltrate nicht mehr als einfaches Ödem gewertet, sondern als Folge der Beatmung interpretiert und als „Respirator lung“ bezeichnet [3].

Die moderne Geschichte des ARDS beginnt 1967 mit der in Lancet erschienenen Meilensteinarbeit von Ashbaugh, Petty et al., die erstmals die klinische Entität des schweren akuten Lungenschadens als „acute respiratory distress syndrome“ beschreibt. In dieser Arbeit werden die vergleichbaren pathophysiologischen Veränderungen und Verläufe von 12 Patienten im Alter zwischen 19 und 48 Jahren beschrieben, obwohl ganz unterschiedliche akute Erkrankungen und Ereignisse wie Pankreatitis, Unfälle, Beinahe-Ertrinken oder Aspirationen zu dem schweren Lungenschaden geführt haben. Auch wenn Petty und Ashbaugh das Krankheitsbild später in Abgrenzung zu dem sehr ähnlichen pathologischen Bild des Syndroms der unreifen Lunge beim Frühgeborenen, dem Infant respiratory distress syndrome (IRDS), als Adult respiratory distress syndrome beschrieben haben [4], hat sich heute die ursprüngliche Bezeichnung Acute respiratory distress syndrome etabliert.

Lange Jahre bestanden doch erheblich unterschiedliche Vorstellungen von dem Krankheitsbild, sodass es sehr schwer war, die klinischen und wissenschaftlichen Beobachtungen aus den unterschiedlichen Untersuchungen zu vergleichen und zu interpretieren.

In ersten Schritten zu einem besseren Verständnis der Erkrankung definierten Murry et al. 1988 die Schwere des Krankheitsbildes nach einem Score-System, das die Items Oxygenation, PEEPDruck, Compliance und die radiologischen Befunde umfasst [5].
Tab. 1 Definition des Acute Lung Injury (ALI) und des Acute Respiratory Distress Syndrome ARDS nach der ARDS-Konsensuskonferenz [6].

\section{Definition ALI/ARDS}

ALI

$\mathrm{PaO}_{2} / \mathrm{FiO}_{2} \leq 300 \mathrm{mmHg}$ unabhängig vom PEEP-Niveau

ARDS:

$\mathrm{PaO}_{2} / \mathrm{FiO}_{2} \leq 200 \mathrm{mmHg}$ unabhängig vom PEEP-Niveau

bilaterale Infiltrate im a. p. Röntgenthorax

Ausschluss Volumenüberladung und/oder Herzinsuffizienz

(PAWP $\leq 18 \mathrm{mmHg}$ )

American-European Consensus Conference on ARDS, AJRCCM 1994; 149: $818-824$

1992 entwickelte dann eine Amerikanisch-Europäische Konsensuskonferenz erstmalig eine standardisierte Definition des ARDS und erweiterte die Entität um den Begriff Acute Lung Injury (ALI) [6]. Nach der Vorstellung der Expertenrunde der Konsensuskonferenz wird ALI als ein Syndrom einer schweren Entzündung der Lunge mit erhöhter Permeabilität verstanden, mit klinischen, radiologischen und physiologischen Befunden, die nicht durch eine kardiale Erkrankung erklärt werden können, häufig in Assoziation mit den Bildern einer schweren Sepsis, Aspiration, Pneumonie, Trauma, akuten Pankreatitis oder anderen Erkrankungen. Das ADRS ist demnach eine Unterform der akuten Lungenschädigung (ALI) mit einer schwereren Gasaustauschstörung ( $\bullet$ Tab. 1).

Histopathologisch ist die Erkrankung gekennzeichnet durch einen diffusen Alveolarschaden mit Schädigung der Pneumozyten Typ 1, Proliferation der Pneumozyten Typ 2, Surfactantstörung und -verlust, Alveolarödem, kapillärer Leakage sowie Infiltration durch Granulozyten und Lymphozyten. Die pathophysiologische Folgen sind eine schwere Gasaustauschstörung, hohes Shuntvolumen, pulmonale Hypertonie, Complianceverlust und eine erhöhte Atemarbeit. In der Regel kann die respiratorische Störung nicht kompensiert werden und der Patient muss maschinell beatmet werden [7] ( $\bullet$ Abb. 1).

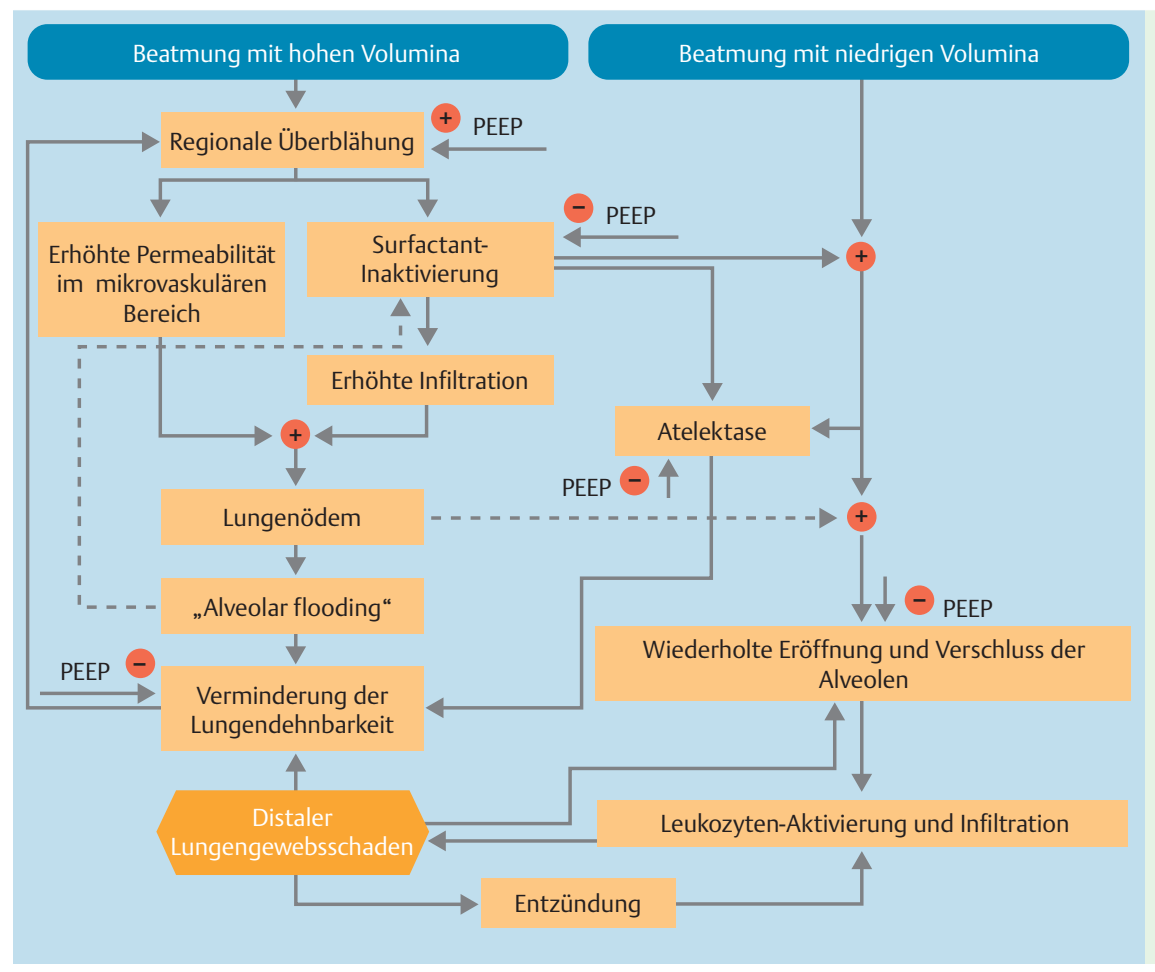

Abb. 1 Pathophysiologie des ventilatorinduzierten Lungenschadens - Auswirkungen des PEEP und der protektiven Beatmung. 


\section{Beatmung bei ARDS}

Die Beatmung steht somit auch im Mittelpunkt der therapeutischen Intervention, sie ist die entscheidende Maßnahme, damit der Patient überleben kann. Allerdings stand die maschinelle Beatmung mit positivem Druck schon sehr früh in dem Verdacht, die Lunge zu schädigen - eine Überlegung, die sich im Laufe der Jahre bestätigte und zu einer sehr differenzierten Betrachtung und Konzeption der Beatmung geführt hat, insbesondere auch weil andere Interventionen wie Surfactantapplikation oder Immunmodulation keinen nachweisbaren Effekt zeigten.

Der beatmende (Intensiv-)Mediziner muss sich bewusst sein, dass die Kraft, die er durch Einstellungen am digital kontrollierten Beatmungsgerät generiert, auf ein für mechanische Reize empfindliches Gewebe einwirkt. Der Beatmungsschaden (ventilator induced lung injury) lässt sich differenzieren in das grobe Zerreißen von Lungenstrukturen mit dem Eindringen von Luft in sonst nicht luftgefüllte Räume, was als Barotrauma bezeichnet wird. Zelluläre Folgen, die sich als inflammatorische Reaktion in der Lunge zusammenfassen lassen, werden als Biotrauma subsummiert. Das Biotrauma kann noch weiter getrennt werden in den Anteil, der durch Überdehnung von Zellen entsteht, was auch als Volutrauma oder als ,high volume lung injury“ bezeichnet wird. Der zweite Anteil wird auch als Atelectrauma oder „low lung volume injury“ bezeichnet und entsteht durch Scherund Zugkräfte bei Kollaps/Wiedereröffnung. Die entstehenden Scherkräfte können das Surfactantsystem schädigen und zu einer vermehrten Permeabilität führen. Wichtig ist die Erkenntnis, dass bei vorgeschädigten Lungen und besonders bei einem ARDS (Acute Respiratory Distress Syndrome) der Grad der Expansion der Alveolen und die Rekrutierbarkeit während der Inflation nicht mehr uniform, sondern inhomogen sind. Je größer der Anteil der Einheiten ist, welche während der Inflation nicht eröffnet werden können, desto kleiner ist der Anteil der Einheiten, welche das Tidalvolumen aufnehmen müssen. Somit steigt dort die Gefahr der Überdehnung durch ein relativ zu großes Volumen. Dieser Effekt wird aufgrund der Größe der verbleibenden, funktionell intakten Lunge auch als der „baby lung effect“ bezeichnet. Die beatmungsassoziierte Schädigung belastet jedoch nicht nur die Lunge alleine, sondern stellt infolge der induzierten Entzündungsreaktionen einen Risikofaktor für die Entwicklung eines Multiorganversagens dar.

\section{ARDS-|ALI-Beatmung heißt heute „protektiv beatmen“ $\nabla$}

Seit Beginn der 90er-Jahre wurden Beatmungskonzepte entwickelt, die eine Überdehnung der Lungen vermeiden. Eine solche, sogenannte lungenprotektive Beatmung sollte nicht nur auf die Aufrechterhaltung der Oxygenierung, sondern auch auf eine Vermeidung beatmungsinduzierter Schäden ausgerichtet sein. Die Vermeidung alveolärer Überdehnung (Volutrauma, „high volume lung injury“) durch niedrige Tidalvolumina oder spezielle Beatmungsmuster (Hochfrequenzventilation) ist dabei ein wesentlicher Aspekt des neuen Beatmungskonzepts [8-11].

Die entscheidende Arbeit, die als die Meilensteinarbeit in der Geschichte der Beatmungsmedizin bezeichnet wird, ist die ARDSNetwork-Studie zur Wertigkeit der Ventilation mit kleinen Atemzugvolumina [9]. In dieser Untersuchung, in die 861 Patienten eingeschlossen wurden, konnte gezeigt werden, dass ein niedriges Atemzugvolumen protektiv ist und die Lunge vor mechanisch induzierter Lungenschädigung schützt. Das Tidalvolumen wurde auf ein aus Körpergröße und Geschlecht errechnetes Normgewicht bezogen. Verglichen wurde die Beatmung mit einem Atemzugvolumen von 6,2 gegen $11,8 \mathrm{ml} / \mathrm{kg}$. Die Mortalität unter dem Beatmungsregime mit niedrigem Tidalvolumen war mit 31,0\% signifikant niedriger als unter dem konventionellen Beatmungsregime mit 39,8\%. Dies entspricht einer absoluten Reduktion der Sterblichkeit um knapp 9\% und einer relativen um 21\% - das wurde alleine durch eine Änderung der Beatmungseinstellungen erreicht.

Diese Arbeit hat zu einem Paradigmenwechsel in der Beatmung geführt - Ziel ist heute so wenig mechanische Unterstützung wie nur möglich. Dies beinhaltet nicht nur eine schonende Beatmung mit wenig Volumen und wenig Druck, sondern auch eine möglichst kurze Beatmungszeit und eine frühzeitige Spontanatmung. Die Entwicklungen neuer, reduzierter Sedierungskonzepte [12,13], wie letztlich auch die zunehmende Anwendung extrakorporaler Gasaustauschsysteme (ECMO, ILA; NovaLung) oder der Hochfrequenzoszillationsventilation (HFOV), sind direkte oder indirekte Folgen der Ergebnisse des Low-tidalvolume Konzeptes [14-17]. Gerade die extrakorporalen Systeme werden in den nächsten Jahren mit der Entwicklung leistungsstarker, aber kleiner werdender und leichter zu bedienender Systeme zu einer entscheidenden Erweiterung der Beatmungsmedizin führen.

\section{Epidemiologie und Inzidenz \\ $\nabla$}

Die Inzidenz des ARDS kann auch heute nur abgeschätzt werden - verlässliche Zahlen sind nicht bekannt und liegen zwischen 15000 und 200000 Fällen/Jahr in den USA [18]. Für Deutschland existieren keine entsprechenden Schätzungen. Die Arbeit von Luhr et al. verdeutlicht das Problem. In dieser Untersuchung fanden sich für jeden ARDS-Fall zehn weitere Fälle mit hypoxämischer respiratorischer Insuffizienz. Über diese Gruppe ist wenig bekannt, hinsichtlich Ursache, klinischem Verlauf und Outcome [19] ( $\odot$ Abb. 2).

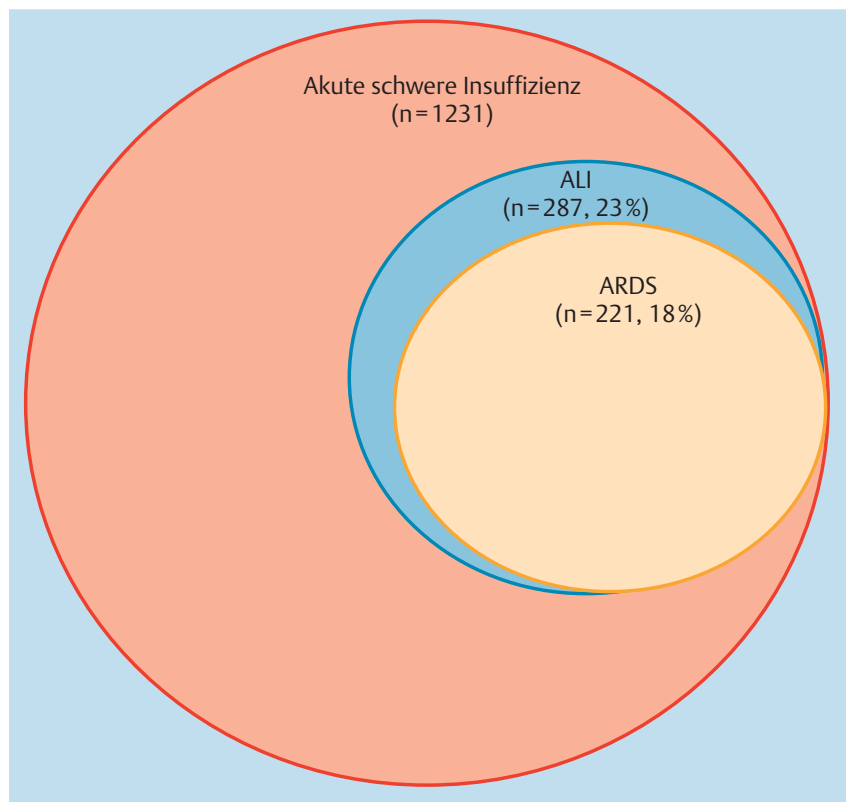

Abb. 2 ARDS- und ALI-Patienten umfassen nur einen kleinen Teil aller Patienten mit akuter respiratorischer Insuffizienz [19]. 


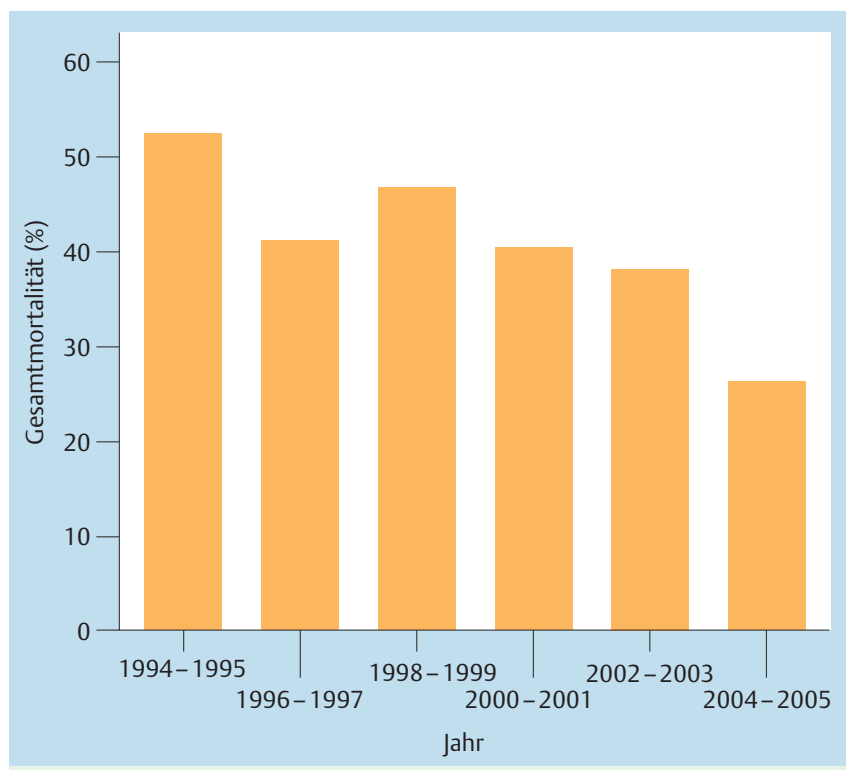

Abb. 3 Veränderung der gepoolten ARDS-Mortalität aus 72 Studien [21].

Eine neuerliche Studie aus Irland [20] erfasste in einem prospektiven Ansatz die Epidemiologie und das Management von Patienten mit ALI und ARDS. Diese Studie lief über zehn Wochen an 14 allgemeinen Intensivstationen. In zehn Wochen wurden 1029 Aufnahmen prospektiv erfasst, 728 Patienten wurden invasiv beatmet. 196 Patienten (19\% der Gesamtaufnahmen) hatten ein ALI bzw. ARDS. Bei 141 von diesen (72\%) war die Diagnose ALI/ ARDS bei der Aufnahme und bei 55 (28\%) im Verlauf der Erkrankung gestellt worden. Das mittlere Alter war $58 \pm 17$ Jahre, $62 \%$ waren Männer. Die häufigste Ursache für das ARDS war mit $50 \%$ die Pneumonie und mit 26\% die nicht pulmonal ausgelöste Sepsis.

\section{Mortalität}

$\nabla$

Die Mortalität des ARDS ist weiterhin sehr hoch. Trotzdem kann von einer Verbesserung des Überlebens in den letzten Jahren ausgegangen werden.

In einer systematischen Analyse der ALI/ARDS-Literatur der Jahre 1994-2005 [21] wurden in 72 auswertbaren Studien insgesamt 11426 Patienten erfasst ( Abb. 3) Die Mortalitätsrate variierte zwischen 15 und 72\%, im Mittel lag sie bei 43\%. Dabei zeigte sich eine signifikant lineare Reduktion der Gesamtmortalität um 1,1\% pro Jahr über den analysierten Zeitraum von 1994 bis 2006. Diese erfreuliche Abnahme der Sterblichkeit kann sicher auf verbesserte Therapiekonzepte zurückgeführt werden, erfordert jedoch den Einsatz von erheblichen Ressourcen im klinischen Bereich.

\section{Beatmungskompetenz - Wer kann besser beatmen? $\nabla$}

Die Beatmungsmedizin hat in den letzten Jahren zunehmend an Bedeutung gewonnen. Zunehmend wird dabei auch die Frage gestellt, wer bzw. welche Fachgruppe die höchste Kompetenz für die Beatmung besitzt.

In einer Untersuchung von Kahn et al. [22] wurde der Frage nachgegangen, inwieweit spezialisierte Intensivstationen eine potenzielle Verbesserung der Beatmung und damit ein verbes- sertes Outcome bieten können. In einer retrospektiven Untersuchung von 1809576 Beatmungspatienten in 8 Staaten der USA, welche $42 \%$ der gesamten Population in den USA erfasst, wurde das Outcome in Kliniken mit vielen und mit wenigen Beatmungsfällen verglichen. Es zeigte sich eindeutig ein Überlebensvorteil für Patienten in Häusern mit einer hohen Zahl von beatmeten Patienten. Rein rechnerisch könnten durch eine Verlegung von Patienten in Zentren mit hoher Beatmungskompetenz 4720 Leben pro Jahr gerettet werden. Dies bedeutet ein Number-to-treat-Verhältnis von 1:15,7. In einer anderen Studie konnte nachgewiesen werden, dass im Vergleich zu Chirurgen die von Pneumologen behandelten ARDS-Patienten ein besseres Outcome zeigten [23].

ALI und ARDS stellen eine der jüngeren Erkrankungen der Lunge dar - erst die modernen Methoden der Medizin, insbesondere der Beatmungsmedizin haben ermöglicht, dass Patienten einen schweren akuten Lungenschaden überleben können. Dabei führten die pathophysiologischen und ätiologischen Erkenntnisse der letzten Jahrzehnte zu der Entwicklung von strukturierten Beatmungskonzepten. Die moderne Pneumologie ist hier im besonderen Maße gefordert und kann aufgrund der breiten Erfahrung in allen Bereichen der Erkrankungen der Atmungsorgane einen wesentlichen Beitrag liefern. Insbesondere die Entwicklung der extrakorporalen Gasaustauschsysteme, aber auch der Systeme zur physiologischeren Beatmungssteuerung, wie die NAVA-Methode (neurally adjusted ventilatory assist) [24], mit der es möglich ist, durch Abgreifen des elektrischen Aktivitätspotenzials des Zwerchfells die Beatmung zu regulieren, zeigen eine spannende und herausfordernde Entwicklung für das Fach Pneumologie und Intensivmedizin auf.

\section{Interessenkonflikte \\ $\nabla$}

Der Autor gibt an, dass kein Interessenkonflikt besteht.

\section{Literatur}

1 Laenenec $R$. A treatise on the diseases of the chest. Birmingham, AL: Classics of Medicine Library, 1979

2 Ibsen B. The anaesthetist's viewpoint on the treatment of respiratory complications in poliomyelitis during the epidemic in Copenhagen, 1952. Proc R Soc Med 1954; 47: 72 - 74

3 Respiratory lung syndrom. Minn Med 1967; 50: 1693 - 1705

4 Petty TL, Ashbaugh DG. The adult respiratory distress syndrome. Clinical features, factors influencing prognosis and principles of management. Chest 1971; 60: 233-239

5 Murray JF, Matthay MA, Luce JM et al. An expanded definition of the adult respiratory distress syndrome. Am Rev Respir Dis 1988; 138: $720-723$

6 Bernard GR, Artigas A, Brigham KL et al. The american-european consensus conference on ards. Definitions, mechanisms, relevant outcomes, and clinical trial coordination. Am J Respir Crit Care Med 1994; 149: $818-824$

7 Ware LB, Matthay MA. The acute respiratory distress syndrome. N Engl J Med 2000; 342: 1334 -1349

8 Villar J, Kacmarek RM, Perez-Mendez L et al. A high positive end-expiratory pressure, low tidal volume ventilatory strategy improves outcome in persistent acute respiratory distress syndrome: A randomized, controlled trial. Crit Care Med 2006; 34: 1311-1318

9 The ARDS-Network. Ventilation with lower tidal volumes as compared with traditional tidal volumes for acute lung injury and the acute respiratory distress syndrome. The acute respiratory distress syndrome network. N Engl J Med 2000; 342: $1301-1308$

10 Amato MB, Barbas CS, Medeiros DM et al. Effect of a protective-ventilation strategy on mortality in the acute respiratory distress syndrome. N Engl J Med 1998; 338: 347-354 
11 David M, Weiler N, Heinrichs W et al. High-frequency oscillatory ventilation in adult acute respiratory distress syndrome. Intensive Care Med 2003; 29: $1656-1665$

12 Kress JP, Pohlman AS, O'Connor MF et al. Daily interruption of sedative infusions in critically ill patients undergoing mechanical ventilation. $\mathrm{N}$ Engl J Med 2000; 342: 1471 - 1477

13 Girard TD, Kress JP, Fuchs BD et al. Efficacy and safety of a paired sedation and ventilator weaning protocol for mechanically ventilated patients in intensive care (awakening and breathing controlled trial): A randomised controlled trial. Lancet 2008; 371: 126 - 134

14 Dembinski $R$, Kuhlen $R$. Extracorporeal gas exchange in acute lung injury: Step by step towards expanded indications? Crit Care 2010; 14: 116

15 Laudi S, Busch T, Kaisers U. Extracorporeal membrane oxygenation for ards due to 2009 influenza a(h1n1). JAMA 2010; 303: 941; author reply 942

16 Peek GJ, Mugford M, Tiruvoipati R et al. Efficacy and economic assessment of conventional ventilatory support versus extracorporeal membrane oxygenation for severe adult respiratory failure (cesar): A multicentre randomised controlled trial. Lancet 2009; 374: 1351 - 1363

17 Lubnow M, Luchner A, Philipp A et al. Combination of high frequency oscillatory ventilation and interventional lung assist in severe acute respiratory distress syndrome. J Crit Care 2010; epub
18 Rubenfeld GD. Epidemiology of acute lung injury. Crit Care Med 2003; 31: S276-S284

19 Luhr OR, Antonsen K, Karlsson $M$ et al. Incidence and mortality after acute respiratory failure and acute respiratory distress syndrome in sweden, denmark, and iceland. The arf study group. Am J Respir Crit Care Med 1999; 159: 1849 - 1861

20 Irish Critical Care Trials Group. Acute lung injury and the acute respiratory distress syndrome in ireland: A prospective audit of epidemiology and management. Crit Care 2008; 12: R30

21 Zambon M, Vincent JL. Mortality rates for patients with acute lung injury/ards have decreased over time. Chest 2008; 133: 1120-1127

22 Kahn JM, Linde-Zwirble WT, Wunsch $\mathrm{H}$ et al. Potential value of regionalized intensive care for mechanically ventilated medical patients. Am J Respir Crit Care Med 2008; 177: 285-291

23 Badin S, Gress TW, Munn N et al. Mechanical ventilation management by pulmonologists and surgeons in patients with adult respiratory distress syndrome. Am J Med Sci 2007; 334: 155-159

24 Sinderby C, BeckJ, Spahija J et al. Inspiratory muscle unloading by neurally adjusted ventilatory assist during maximal inspiratory efforts in healthy subjects. Chest 2007; 131: 711-717 